\title{
Uncommon Clinical Manifestation of Cerebral Arteriovenous Malformation in Children
}

\author{
Ana Belen Ariza Jimeneza ${ }^{1^{*}}$, Maria Jose Pelaez Canterob ${ }^{2}$ and Jacinto Martinez Antonc ${ }^{3}$ \\ ${ }^{1}$ Pediatrician, Santa Barbara Hospital, Spain \\ ${ }^{2}$ Pediatrician, Malaga Children's Hospital, Spain \\ ${ }^{3}$ Neuropediatrician, Malaga Children's Hospital, Spain
}

*Corresponding author: Ana Belén Ariza Jiménez, C / Eugenio Gross, 50 7C. Málaga, Spain, Tel: 34666154496; E-mail: micodemas@hotmail.com

Rec date: Feb 28, 2016; Acc date: Apr 25, 2016; Pub date: Apr 30, 2016

Copyright: (c) 2016 Jiméneza ABA, et al. This is an open-access article distributed under the terms of the Creative Commons Attribution License, which permits unrestricted use, distribution, and reproduction in any medium, provided the original author and source are credited.

\begin{abstract}
Seizures are the second presentation of cerebral vascular malformations, while the first cause is bleeding. However, there are few publications about it, so we describe a case in which the photosensitive epilepsy is the first clinical manifestation of an occipital arteriovenous malformation, for scientific interest and clinical knowledge about it. Our patient was diagnosed by magnetic resonance imaging and arteriography, and he was treated with embolizations with good outcome.
\end{abstract}

Keywords: Reflex epilepsy; Photosensitive epilepsy; Arteriovenous malformation; Embolization; Image diagnosis

\section{Learning Points}

- Photosensitive epilepsy could be an uncommon presentation of arteriovenous malformation

- The main diagnostic is done by image, which has a very important role.

- Embolization is an effective treatment in this case

\section{Uncommon Clinical Manifestation of Cerebral Arteriovenous Malformation}

Arteriovenous malformations (AVM) are congenital lesions due to an embryogenic failure during vascular differentiation [1]. According to literature, brain AVM is less often in children than in adults [2]. The most frequent clinical presentation is bleeding, while epilepsy is the second one. Size [3] and temporal localization increase seizures risk [4].

Although, the association between photosensitive epilepsy and arteriovenous malformations is not well described in literature, there are three pathophysiological mechanisms which could explain its relation, such as theft phenomenon related to hypoxia, arteriovenous malformation brain gliosis caused by bleeding and hemosiderin deposition, and AVM's epileptogenic role [5].

We present a 10 years old boy with an episode of disconnection plus generalized hypertonia, clonic movements, drooling and trismus, while he was jumping on an inflatable playground castle with continuous changes from sunlight to shadow. This episode finished spontaneously without medication in about 5 minutes, although it associated a postictal period of 10 minutes.

On the other hand, neurological examination and cranial Computed Tomography (CT) were normal. Nevertheless, on vigil Electroencephalogram (EEG), we could see a discharge of generalized spike-waves, which were induced by 21 flashes per second, and on EEG in sleep deprivation, we could observe irregular slow waves and spikes with intermittent light stimulation from 12 to 18 flashes per second. EEG without light stimulation was normal.

Cranial magnetic resonance imaging (MRI) revealed an AVM in middle and posterior left cerebral arteries (Figure 1a).

Arteriography showed a $6 \times 4.5 \times 4 \mathrm{~cm}$ left occipito-parietal MAV, which made a non-homogeneous nidus with arterial branches from middle and posterior left cerebral arteries (Figure 1b).

He was programmed to embolization in several times. He needed anticonvulsants because of several epileptic crisis and contralateral motor deficit after embolizations (Figure 1b).

Nowadays, he is stable with an important improvement showed in arteriography (Figure 1b) and normal neurological examination.

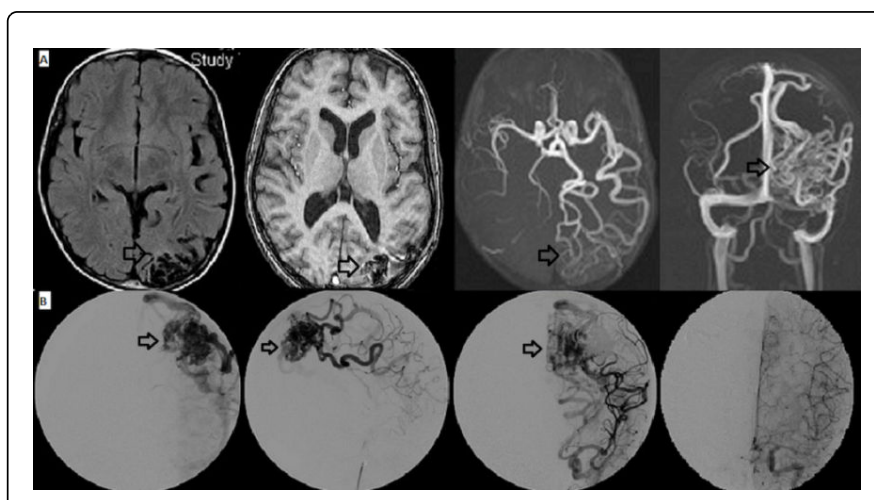

Figure 1: a) Arteriovenous malformation before treatment on magnetic resonance, and arteriography in venous (right) and arterial phase (left) b) Evolution during embolizations. In the last one, malformation has dissapeared.

Only $15 \%$ of arteriovenous malformations are presented as chronic seizure disorder, the same as in our case [1]. 
Citation: Jimeneza ABA, Canterob MJB, Antonc JM (2016) Uncommon Clinical Manifestation of Cerebral Arteriovenous Malformation in Children. J Clin Case Rep 6: 771. doi:0.4172/2165-7920.1000771

Page 2 of 2

According to our case, we should consider cranial MRI with and without gadolinium, and magnetic resonance angiography, as adequate diagnosis methods for photosensitive epilepsy. Nevertheless, we have treated our patient with anticonvulsants; it is actually a matter of controversy [5]. Nowadays, resection of vascular injury is a recommended treatment for arteriovenous malformation with bleeding risk [5]. It is important to highlight that Interleukin-6 is a good predictor of bleeding, but we do not have it available in our hospital [2].

Success of treatment depends on AVM's location and size, hemodynamic characteristics, medical and general condition of the patient, and treatment method [5].

Available treatments for arteriovenous malformation have increased with advances in technology. Nowadays, they include microsurgical resection, endovascular embolization, radiosurgery and any combination of these 5; therefore, we used endovascular embolization with good results.

\section{Conclusion}

In conclusion, it is important to consider AVM as a possible cause of photosensitive epilepsy, in which MRI and arteriography are the best diagnostic and following methods. An adequate treatment is vascular embolization, and the use of anticonvulsants remains controversy at this moment.

\section{References}

1. Laakso A, Hernesniemi J (2012) Arteriovenous malformations: epidemiology and clinical presentation. Neurosurg Clin N Am 23: 1-6.

2. Li X, Wang R, Wang X, Xue X, Ran D, et al. (2013) Relevance of IL-6 and MMP-9 to cerebral arteriovenous malformation and hemorrhage. Mol Med Rep 7: 1261-1266.

3. Sturiale CL, Rigante L, Puca A, Di Lella G, Albanese A, et al. (2013) Angioarchitectural features of brain arteriovenous malformations associated with seizures: a single center retrospective series. Eur J Neurol 20: 849-55.

4. Galletti F, Costa C, Cupini LM, Eusebi P, Hamam M, et al. (2014) Brain arteriovenous malformations and seizures: an Italian study. J Neurol Neurosurg Psychiatry 85: 284-288.

5. Brown RD (2008) Unruptured brain AVMs: to treat or not to treat. Lancet Neurol 7: 195. 